\title{
Temporal Lobe
}

National Cancer Institute

\section{Source}

National Cancer Institute. Temporal Lobe. NCI Thesaurus. Code C12353.

One of the cerebral lobes. It is located inferior to the frontal and parietal lobes and anterior to the occipital lobe. 